\title{
ORIGIN OF BOTH GREAT VESSELS FROM THE ARTERIAL VENTRICLE: A COMPLEX WITH VENTRICULAR INVERSION*
}

\author{
BY
HERBERT D. RUTTENBERG $\dagger$, RAY C. ANDERSON, LARRY P. ELLIOTT, AND JESSE E. EDWARDS

From the Departments of Pediatrics, Radiology, and Pathology, the University of Minnesota, Minneapolis, Minnesota U.S.A., and the Department of Pathology, The Charles T. Miller Hospital, St. Paul, Minnesota, U.S.A.

Received December 9, 1963

While origin of both great vessels from the right ventricle (Neufeld, DuShane, and Edwards, 1961 ; Neufeld et al., 1961) is an uncommon form of transposition, from the left-sided ventricle it is almost unknown. One author stated that, theoretically, the malformation from the left ventricle does not exist (Grant, 1962). As far as we have been able to discover, only one case (Fragoyannis and Kardalinos, 1962) has been described.

Spitzer (1951) was the first to point out that various forms of transposition can occur in the inverted state. He proposed that the inverted form of complete transposition is corrected transposition wherein the great vessels are transposed and arise from the functionally correct but inverted ventricles. He further stated that the process of bulbo-ventricular inversion may occur in simple transposition of the aorta, i.e. origin of both great vessels from the right ventricle or "double outlet" right ventricle (Witham, 1957), in which case both great vessels would arise from the left-sided (arterial) ventricle. This was the situation found in the case of Fragoyannis and Kardalinos (1962) and in the three presented here.

In this communication the pathological findings in three cases of the very rare malformation, origin of both great vessels from the arterial ventricle, are reported in detail. The relations between this and other forms of transposition of the great vessels are commented upon.

Although reference to these three cases was made in a previous report on congenital cardiac disease with asplenia (Ruttenberg et al., 1964), limitation of space precluded a detailed description of them and emphasis of this condition as a developmental entity.

The pathological findings will be discussed first, followed by a brief review of the clinical findings. With the exception of selective angiocardiography, the clinical findings were of limited value in the diagnosis of this condition.

\section{DEFINITIONS}

For a common understanding it is necessary to define the terms that describe the location, internal anatomical architecture, and hæmodynamic characteristics of the ventricles.

The terms "left-sided" and "right-sided" refer to the location of the ventricles. When the process of inversion has occurred, the inverted ventricle has the anatomical internal architecture of

* This study was supported by Research Grant No. HE 5694 and Research Training Grant No. HTS-5570 from the National Heart Institute, National Institutes of Health, United States Public Health Service.

† Dr. Ruttenberg was a Postdoctoral Fellow (HF 13, 715), National Heart Institute, National Institutes of Health, United States Public Health Service. 
the contralateral ventricle of the normal. For example, in the situs solitus heart with ventricular inversion, the left-sided ventricle is highly trabeculated and possesses a crista supraventricularis and an infundibulum. These are anatomical characteristics of the normal right ventricle. Moreover, when ventricular inversion is present, the right-sided ventricle is smooth-walled, a characteristic feature of the left ventricle of normal hearts.

To carry this example further, the inverted left-sided ventricle is the "arterial ventricle" hamodynamically, since it is connected with the normally-positioned left atrium. Conversely, the inverted right-sided ventricle is the "venous ventricle" since it connects with the normally-positioned right atrium. The hæmodynamic terms of "venous" or "arterial" ventricle are used in the cases described here even though associated venous anomalies have produced abnormal hæmodynamic states in the atrial portions of the hearts.

\section{Pathological Anatomy}

Cases 1 and $2^{*}$. The pathological anatomy in the first two cases was similar and will, therefore, be described together (Fig. 1 and 2). The cardiac malformation in these two was closely related to that seen in corrected transposition of the great vessels.

In each case the cardiac apex pointed toward the right (dextroversion of the ventricles), though the atrial portion of the heart was of situs solitus structure. The interrelation of the great vessels was similar to that found in corrected transposition. The aorta rose anteriorly and ascended above the left basal aspect of the heart, while a hypoplastic pulmonary trunk rose directly posteriorly to the aorta. The coronary arterial pattern was characteristic of that found in corrected transposition: the right coronary artery rose above the right aortic cusp and gave rise to an anterior descending branch; the left coronary artery originated above the posterior cusp and took the course of a left circumflex artery; and the left aortic cusp was non-coronary.

Ventricular inversion was present in each case. The right-sided (venous) ventricle possessed a smoothwalled cavity like that of the left ventricle of normal hearts. The only outlet for this chamber was a large ventricular septal defect, which formed the inferior aspect of a persistent common atrio-ventricular canal. The left-sided (arterial) ventricle was highly trabeculated and possessed a crista supraventricularis like the right ventricle of normal hearts. In Case 1 the ventricles were enlarged and approximately equal in size. In Case 2 the right-sided ventricle was dilated and larger than the left-sided ventricular chamber.

In each case, the aorta originated anteriorly to the crista supraventricularis and a stenotic subpulmonary channel also originated from the left-sided (arterial) ventricle. This channel terminated at a stenotic pulmonary valve which was bicuspid in Case 1 and unicuspid in Case 2. The pulmonary and aortic valves were located at the same horizontal body plane. In neither case was there continuity between atrio-ventricular valvular tissue and the aortic or pulmonary semilunar valvular elements.

In Case 1, the subpulmonary channel originated just inferiorly to the posterior aortic cusp and ascended obliquely in a postero-superior direction through the substance of the crista supraventricularis (Fig. 1C and D). In Case 2, the narrow subpulmonary tract originated medially to the aortic valve and slightly anterior to the septal limb of the crista supraventricularis. This tract also ran obliquely superiorly and posteriorly through the septal limb of the crista (Fig. 2B and D).

In each of these two cases, other associated cardiovascular features were bilateral superior venæ cavæ and absent coronary sinus. In Case 1 there was total anomalous pulmonary venous connexion to the right superior vena cava, while in Case 2 the pulmonary veins were connected to the left (arterial) side of the common atrium by a stenotic common pulmonary vein (mild cor triatriatum).

Case 3. Although some of the pathological features in Case 3 were similar to those in Cases 1 and 2, other features were unusual and require a separate description (Fig. 3).

Included among the anomalies in this case with situs inversus were mirror-image dextrocardia with ventricular inversion, atresia of the right-sided (arterial) atrio-ventricular valve, and origin of both great vessels from the right-sided (arterial) hypoplastic ventricle. The great vessels were transposed and their interrelations were somewhat atypical for corrected transposition. Although the aorta arose anteriorly and to the right, as would be expected for corrected transposition in situs inversus, a hypoplastic pulmonary trunk originated obliquely, posteriorly, and to the right of the aorta (Fig. 3A and C).

* We are indebted to Dr. Werner Kraatz of Saint Paul, Minnesota, for providing the specimen in Case 2. 


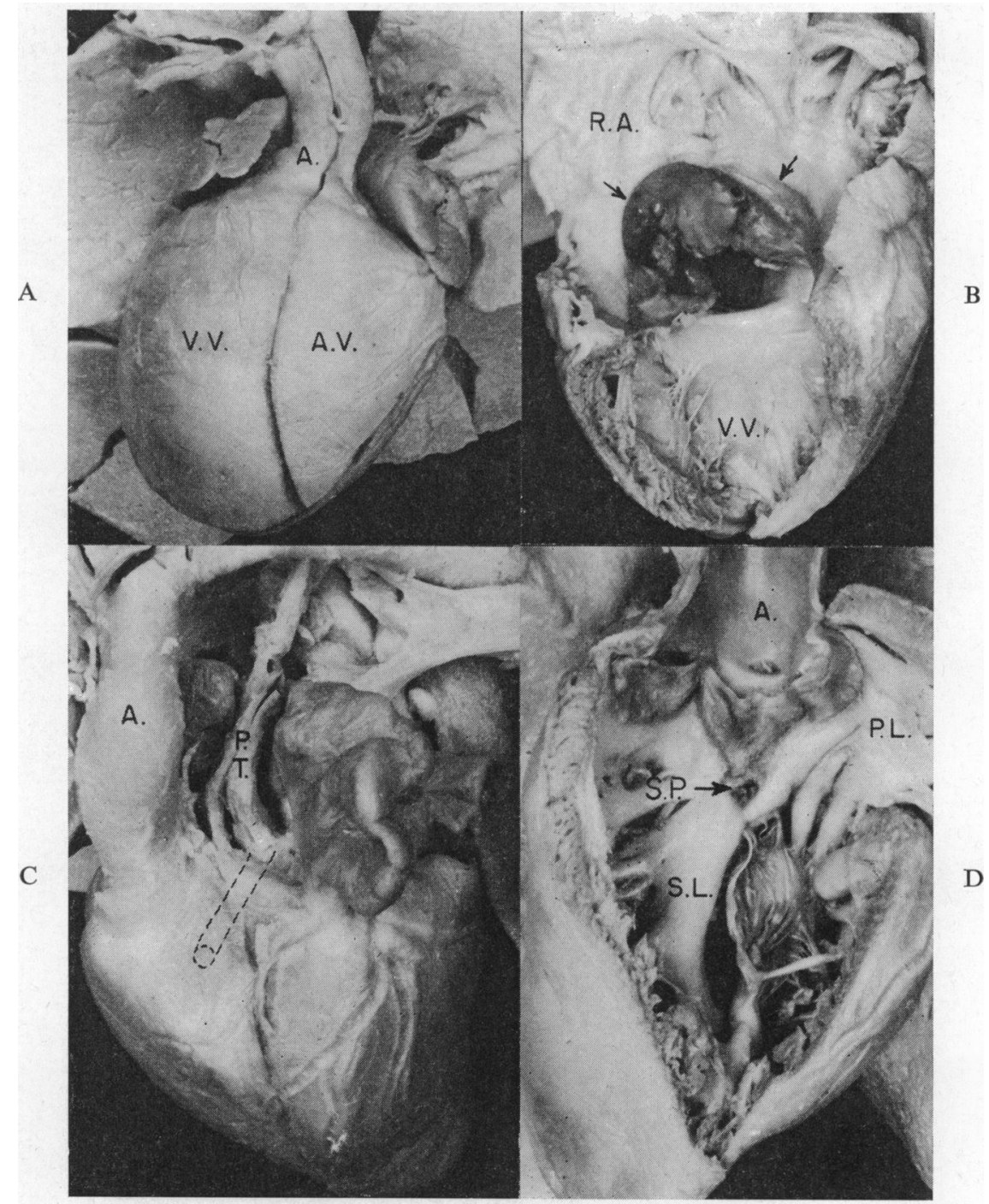

FIG. 1.-Case 1: origin of both great vessels from the left-sided (arterial) ventricle. (A) Anterior view of the heart and great vessels. The cardiac apex is formed by the right-sided venous ventricle (V.V.) and is directed toward the right (dextroversion). The transposed aorta (A.) rises anteriorly above the left-sided arterial ventricle (A.V.). (B) Right lateral interior view of right atrium (R.A.) and inverted venous ventricle (V.V.). Below the fossa ovalis lies a large crescent-shaped defect (arrows) which is part of the persistent common atrio-ventricular canal. The only outlet for this ventricle is through the canal. (C) Left lateral view of the heart and great vessels showing transposed aorta (A.) rising anteriorly to the hypoplastic pulmonary trunk (P.T.). The dotted lines are a diagrammatic representation of the course of the subpulmonary channel. (D) Interior of arterial ventricle. The aorta (A.) originated above the crista supraventricularis (S.L. =septal limb; P.L. = parietal limb). An arrow (S.P.) points to the orifice of the stenotic subpulmonary channel located inferiorly to the posterior aortic cusp.

The left-sided (venous) ventricle was smooth-walled and did not possess a crista supraventricularis (Fig. 3D). In situs inversus this is characteristic of ventricular inversion (Schiebler et al., 1961). The only outlet from this chamber was a large defect in the ventricular septum. Chord-like elements of one leaflet of the left-sided A-V valve passed through the ventricular septal defect into the right-sided (arterial) ventricle 


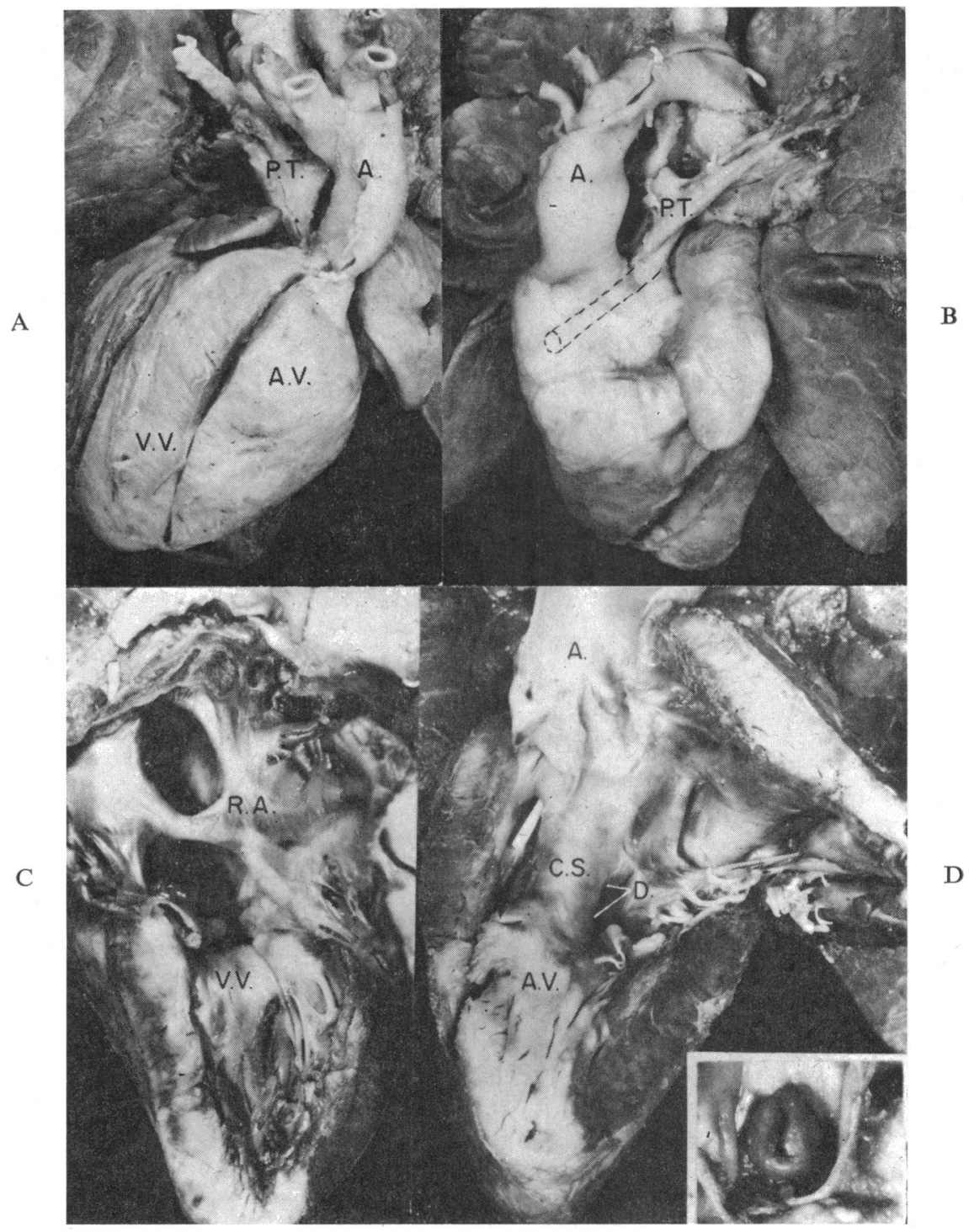

FIG. 2.-Case 2: origin of both great vessels from the left-sided (arterial) ventricle. (A) Anterior view of heart and great vessels. The cardiac apex which is formed by the right-sided venous ventricle (V.V.) is directed toward the right (dextroversion). The transposed aorta (A.) rises anteriorly and to the left above the left-sided arterial ventricle (A.V.). The hypoplastic pulmonary trunk (P.T.) rises posteriorly to the aorta. (B) Left lateral view of heart and great vessels showing transposed aorta (A.) rising directly anteriorly to the hypoplastic pulmonary trunk (P.T.). The dotted lines are a diagrammatic representation of the course of the subpulmonary channel. (C) Right lateral interior view of the venous ventricle (V.V.) showing the smooth septal wall, characteristic of the inverted ventricle. The only outlet of this chamber is the defect formed by the persistent common atrio-ventricular canal. R.A. is right side of the common atrium. (D) Interior of inverted arterial ventricle (A.V.). The aorta (A.) rises anteriorly to the crista supraventricularis (C.S.). The probe is in the stenotic subpulmonary channel which runs obliquely posteriorly and superiorly to the pulmonary valve. A large ventricular septal defect (D.) is bounded anteriorly by the crista supraventricularis (C.S.) and posteriorly by the common atrio-ventricular valvular tissue. Insert. Stenotic unicuspid pulmonary valve from above. 


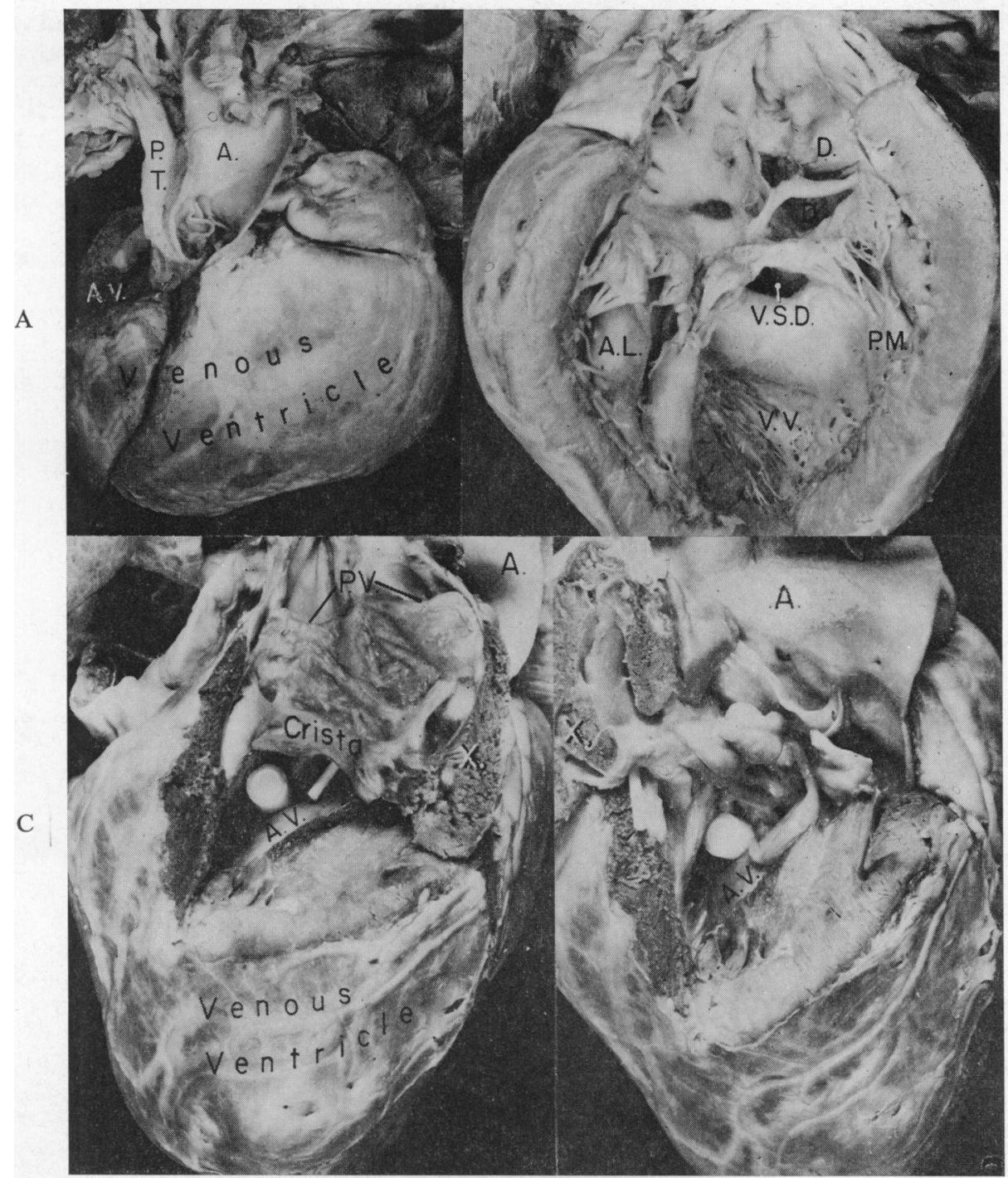

FIG. 3.-Case 3: origin of both great vessels from the right-sided (arterial) ventricle with mirrorimage dextrocardia. (A) Anterior view of heart and great vessels. Transposed aorta (A.) rises anteriorly above the right cardiac border. - Hypoplastic pulmonary trunk (P.T.) is posterior and slightly to the right of the aorta. Both great vessels originate from a hypoplastic right-sided arterial ventricle (A.V.). The left-sided venous ventricle is very large and forms the right-sided apex of the heart. (B) Left lateral interior view of the venous ventricle (V.V.) showing the smooth septal wall which is characteristic of an inverted left-sided venous ventricle in situs inversus. The only outlet for this chamber is a defect (V.S.D.) in the ventricular septum. The only remnant of the atrial septum is a strand of tissue which separates the superior defect (D.) in the area of the foramen ovale from the lower defect (D.). The inverted venous atrio-ventricular valve is mitral in character with an antero-lateral papillary muscle (A.L.) and a postero-medial papillary muscle (P.M.). All systemic and pulmonary venous blood flowed into the venous ventricle because the right atrio-ventricular valve was atretic. (C) and (D) Right lateral interior view of the hypoplastic arterial ventricle (A.V.). The right lateral ventricular wall has been removed. (C) The large probe lies in the ventricular septal defect. A small probe is placed through the aortic valve and is directed toward the anteriorly located aorta (A.). The pulmonary trunk has been opened demonstrating the bicuspid pulmonary valve (P.V.). Between the pulmonary valve and the hypoplastic crista supraventricularis is a stenotic subpulmonary channel made narrow by endocardial fibrous folds. (X. is flap of tissue which was moved posteriorly to obtain illustration in D.) (D) The flap of tissue (X.) has been moved posteriorly as compared to the illustration in C. This exposes the aortic valve and ascending aorta (A.) Atrio-ventricular valvular tissue protrudes through the ventricular septal defect (above the large probe) and is in continuity with the aortic valve. The small probe lies in the subpulmonary channel. 
and were continuous with elements of the aortic valve. This fibrous tissue projected into the arterial ventricle, immediately inferiorly and posteriorly to the aortic valve and possibly caused sub-aortic stenosis (Fig. 3D). In the right-sided (arterial) ventricle, which was hypoplastic, a structure resembling the crista supraventricularis separated the aortic valve antero-medially, from the pulmonary valve postero-laterally (Fig. 3C). The pulmonary valve lay at a slightly higher level than did the aortic valve. There was no continuity between pulmonary valvular and atrio-ventricular valvular elements. A subpulmonary channel was made narrow by the presence of irregular and excessive endocardial fibrous folds. The pulmonary valve was bicuspid and stenotic.

Additional features were bilateral superior venæ cavæ, a common atrium, and absence of the coronary sinus.

\section{Clinical Findings}

In each case there was cyanosis from birth. In Case 1, a female infant who died 16 hours after birth, no special clinical studies had been performed, while in the other cases, electrocardiograms, thoracic radiographs, and angiocardiograms were available for review. These will be discussed in a subsequent section of this report.

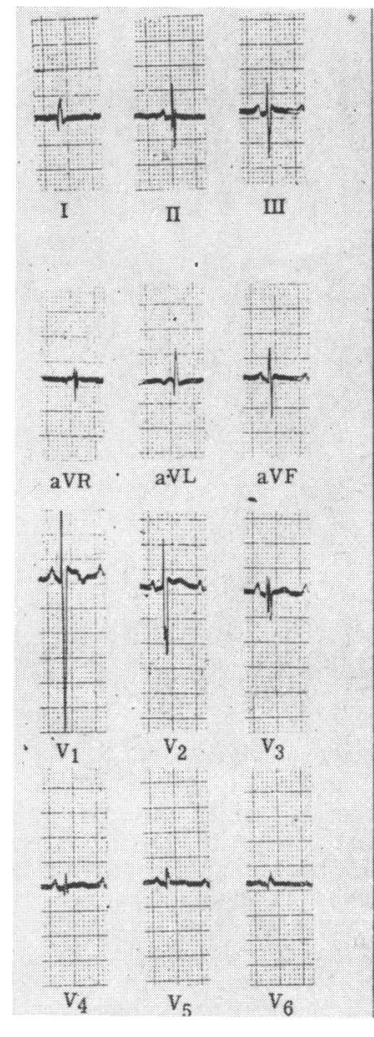

A

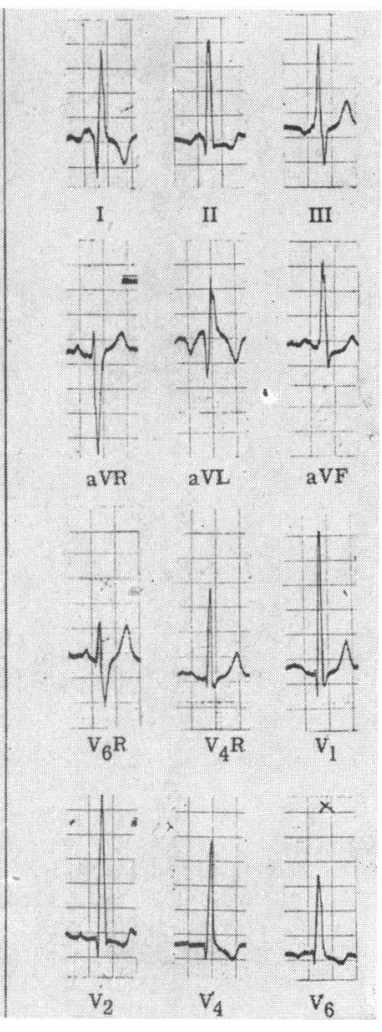

B

- FIG. 4.-Electrocardiograms in Cases 2 and 3. (A) Case 2: origin of both vessels from the left-sided arterial ventricle with dextroversion. There is left axis deviation with a counterclockwise QRS loop in the frontal plane (standard leads). The largest QRS complex in the præcordial leads was located in lead V4R (not shown) and is illustrated in lead V1. The QRS voltages decrease progressively toward leads V6 and V6R (not shown). This pattern is suggestive of dextroversion and of hypertrophy of the right-sided large ventricle. (B) Case 3: origin of both great vessels from the right-sided (arterial) ventricle with mirror-image dextrocardia. After correcting the limb leads for the situs inversus, the QRS axis in the frontal plane is +135 degrees and the QRS loop is inscribed clockwise. The præcordial pattern of $\mathrm{qR}$ in leads V2 through V6 and $\mathrm{rS}$ in lead V6R suggests marked hypertrophy of the left-sided (venous) ventricle. There is, in addition, complete left-sided bundle-branch block. 
The second patient (Case 2) was a boy who died at 2 months of age. Findings in peripheral blood smears suggested asplenia, while the radiological studies suggested the diagnosis of corrected transposition with pulmonary stenosis, ventricular septal defect, and dextrocardia.

The third patient (Case 3 ) died at 4 years of age shortly after a Blalock-Taussig anastomosis had been performed. The clinical studies suggested a diagnosis of cor biloculare (or atrioventricular canal), anomalous pulmonary venous connexion, transposition of the great vessels, and mild pulmonary stenosis with mirror-image dextrocardia. These findings were thought to fit the asplenic syndrome, and the latter possibility was confirmed by the findings in smears of the peripheral blood.

The electrocardiograms in Cases 2 and 3 were helpful in differentiating dextroversion (isolated dextrocardia) from mirror-image dextrocardia. In Case 2 (Fig. 4A), the $P$ axis in the frontal plane was +90 degrees. The $P$ waves were peaked and broad in lead V3 suggesting right and left atrial enlargement. The largest QRS complex in the præcordial leads was at lead V4R (rS) while the QRS complexes in lead V6 (qr) and in lead V6R (rS) were small. This pattern suggests dextroversion of the ventricles and hypertrophy of the right-sided ventricle. Vectorial analysis of the QRS complexes revealed left axis deviation (zero degrees) with a counterclockwise loop in the frontal plane suggesting the presence of an "atrio-ventricular commune" malformation.

In Case 3 (Fig. 4B) the $P$ axis in the frontal plane was +120 degrees; this finding suggested mirrorimage dextrocardia. The $P$ waves were biphasic, tall, and broad in the limb leads, suggesting biatrial enlargement. After correcting the standard limb leads for mirror-image dextrocardia, the mean QRS axis in the frontal plane was +135 degrees and the QRS loop was inscribed clockwise. Marked hypertrophy of the left-sided (venous) ventricle was suggested by the pattern of $\mathrm{qR}$ in leads V2 through V6 (with large $R$ waves) and of $r S$ in lead V6R. In addition, there was complete leftsided bundle-branch block.

Thoracic radiographs were available in Cases 2 and 3. In both the cardiac apex was directed toward the right. In Case 2 (Fig. 5), the cardiac size was normal and the pulmonary vasculature appeared less prominent than normal. There was a prominence in the left side of the superior mediastinum which represented the enlarged ascending aorta. The lower edge of the liver was transverse as is commonly seen in the asplenic syndrome. The barium-filled stomach was seen on the left side, indicating a predominantly situs solitus position of the abdominal organs.

In Case 3 (Fig. 6a and b), the heart was enlarged and the pulmonary vasculature was normal. The convex border of the left superior mediastinum and the indentation of the right border of the barium-filled œsophagus suggested a right aortic arch. Situs inversus of the stomach was present.

Angiocardiograms were performed from the saphenous vein in Case 2, and from the right-sided (arterial) ventricle in Case 3.

Review of the study in Case 2 (Fig. 7) revealed sequential opacification of the inferior vena cava 


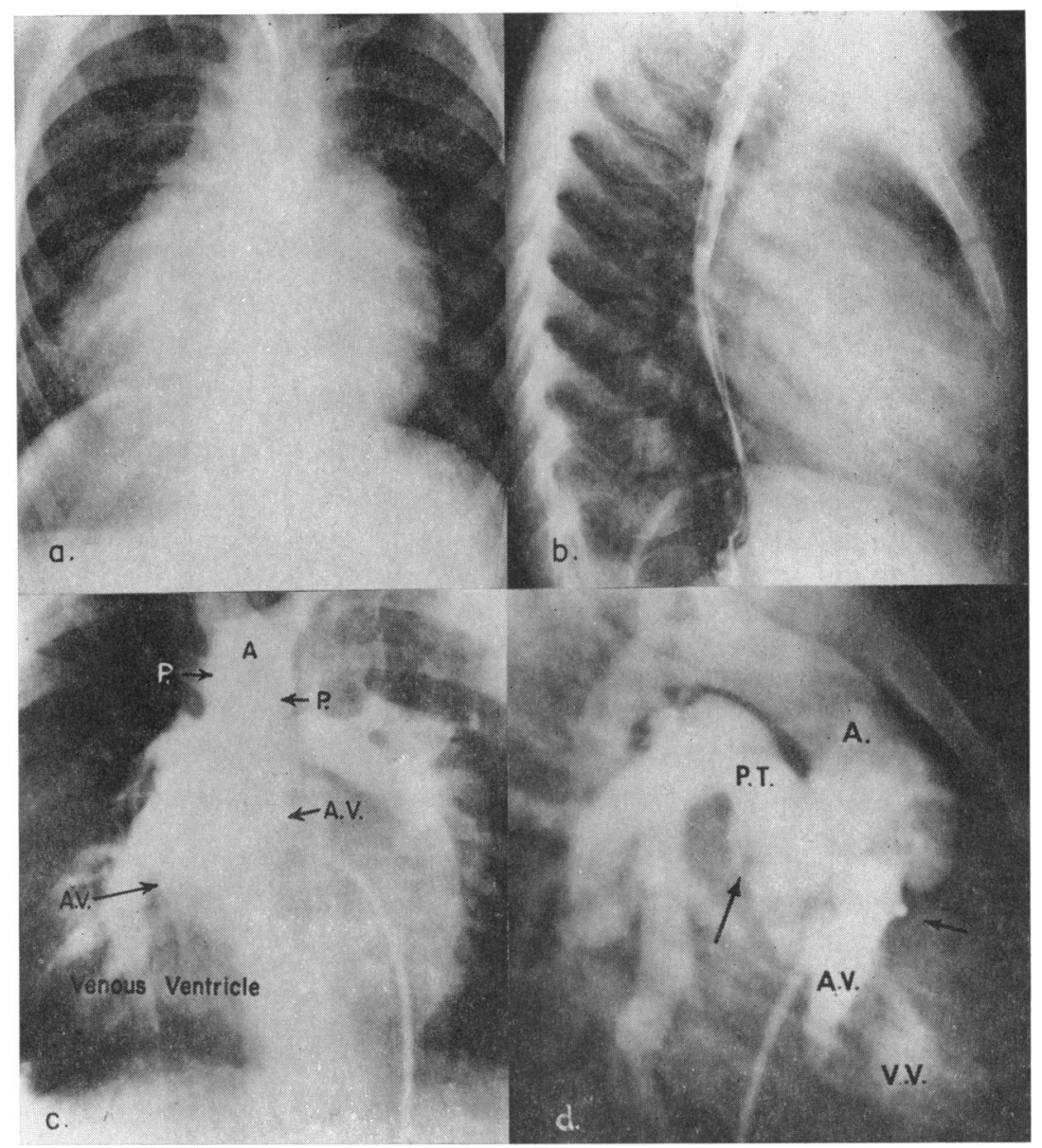

FIG. 6.-Case 3: origin of both great vessels from the right-sided (arterial) ventricle with mirrorimage dextrocardia. (a) Thoracic radiograph, frontal projection, showing that the apex is directed toward the right and there is moderate cardiomegaly. The pulmonary vasculature is normal. The indentation of the right side of the barium-filled œsophagus suggests the presence of a right aortic arch. (b) Lateral projection showing cardiomegaly and atrial enlargement. (c) and (d) Selective angiocardiogram from the rudimentary right-sided (arterial) ventricle. (c) Frontal projection: the small right-sided arterial ventricle (A.V.) is opacified and its borders are marked by lower pair of facing arrows. The pulmonary trunk (between facing arrows $P$.) is located directly behind the aorta (A), and both vessels originated above the arterial ventricle. (d) Lateral projection: there is some regurgitation of contrast material into the left-sided venous ventricle (V.V.). Right arrow points to tip of catheter in the arterial ventricle (A.V.); left arrow points to the pulmonary valve. The aorta (A.) rises anteriorly to the pulmonary trunk (P.T.), and both vessels originate from the small right-sided (arterial) ventricle.

(which was on the right), right and left sides of the common atrium, and of the large right-sided (venous) ventricle. The apex of this ventricle was pointed and lay in the right hemithorax. The aorta, situated anteriorly, opacified next through a large ventricular septal defect. It originated from the antero-basal aspect of the small, anterior, left-sided (arterial) ventricular chamber. Opacification of the pulmonary trunk occurred just before the aorta was opacified. The site of origin of the pulmonary trunk, however, could not be determined. The arrangements of the great vessels and contour of the ventricles suggested the diagnosis of corrected transposition with dextroversion.

In Case 3, during cardiac catheterization, a selective angiocardiogram was performed from the rudimentary right-sided (arterial) ventricle (Fig. $6 \mathrm{c}$ and d). The catheter was passed from the 


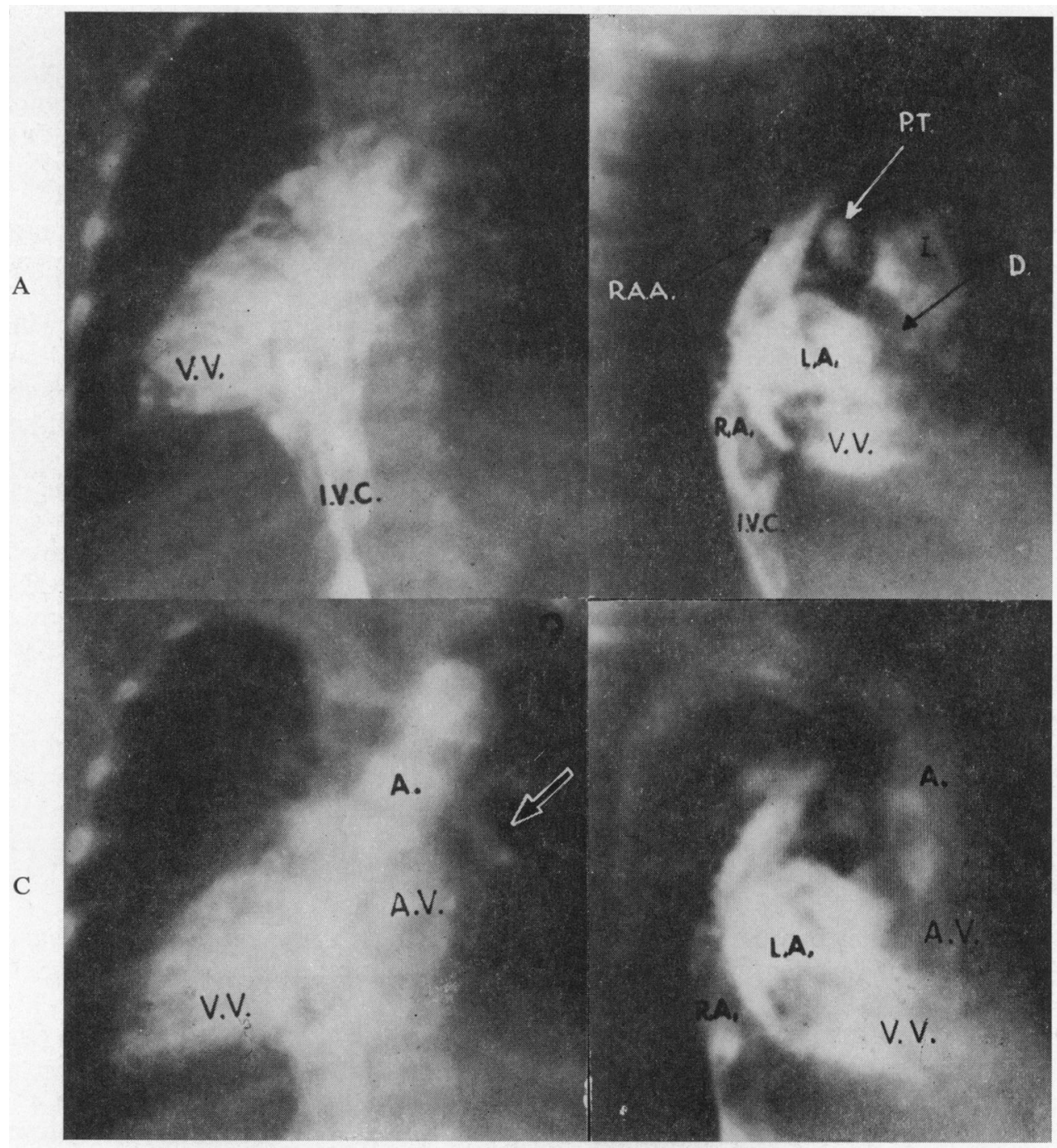

FIG. 7.-Case 2: forward angiogram from saphenous vein. (A) Frontal projection: a large right-sided venous ventricle (V.V.) is opacified; the inferior vena cava (I.V.C.) is located on the right. (B) Lateral projection: companion film of $\mathrm{A}$; there is sequential filling of the inferior vena cava (I.V.C.), right side (R.A.) and left side (L.A.) of the common atrium, the right atrial appendage (R.A.A.), and the right-sided venous ventricle (V.V.). Contrast material passes through a ventricular septal defect (D.) and opacifies the infundibulum (I.) of the left-sided arterial ventricle. A small posterior pulmonary trunk (P.T.) is opacified before the aorta. (C) Frontal projection $0.2 \mathrm{sec}$. after films shown in A and B. The left-sided arterial ventricle (A.V.) is now opacified; the aorta (A.) originated above this chamber. Arrow points to left pulmonary artery. (D) Lateral projection. Companion to film in C. The transposed aorta (A.) arises anteriorly above the arterial ventricle (A.V.).

saphenous vein through the inferior vena cava, which was on the left, into the atrial portion of the heart, then into the huge left-sided ventricle, through the ventricular septal defect, and into the small right-sided ventricle where the injection was made. The study revealed that the tip of the catheter was positioned in a small right-sided (arterial) ventricle from which both great vessels originated. The aorta was located anteriorly and to the right, while the pulmonary trunk rose directly posteriorly to the aorta. The left-sided (venous) ventricle was greatly enlarged, lay anteriorly, and formed the cardiac apex on the right side. 


\section{COMMENT}

The pathological anatomy in each of our cases was intimately related to corrected transposition of the great vessels. In common with corrected transposition, our cases exhibited: (1) inversion of the ventricles and of the coronary arterial pattern; (2) the peculiar interrelation of the great vessels; and (3) the aorta originating from the arterial ventricle anteriorly to the crista supraventricularis (in Cases 1 and 2).

In contrast to the features of corrected transposition, our cases displayed origin of the pulmonary trunk from the arterial ventricle and lack of continuity between A-V valvular tissue and elements of the pulmonary valve.

In Case 3, with mirror-image dextrocardia, there were additional atypical anatomical findings. First, there was direct continuity between aortic and left-sided A-V valvular tissue: this is an extremely unusual arrangement for any type of transposition wherein the aorta rises anteriorly to the pulmonary trunk. Secondly, the aortic valve lay at a slightly lower level than the pulmonary valve.

The three cases in this report represent an entity among cases with transposition of the great vessels. Whether or not it should be considered the inverted form of origin of both great vessels from the right ventricle is an unsettled matter. It is interesting, however, to compare these two anomalies. One may view the condition of origin of both great vessels from the right ventricle as closely related to the normal heart with the exception that the aorta is transposed across the ventricular septum to originate from the right ventricle. One may also envisage a similar process occurring in corrected transposition. In this case, the pulmonary trunk is transposed across the septum, to take origin from the left-sided (arterial) ventricle.

Recently, Grant (1962), presenting a new theory on the morphogenesis of transposition of the great vessels, offered an explanation for the occurrence of "double outlet" right ventricle as a variant of transposition. He stated that there "probably will never be described cases of 'double outlet' left ventricle." It may be assumed that he was referring to origin of both great vessels from a noninverted left ventricle since he did not mention the process of inversion as it may occur with transposition.

The claim of Grant may be valid if one restricts the definition of a left ventricle to that chamber, whatever its position or function, which has the anatomical characteristics of the left ventricle of normal hearts.

The term left ventricle may be also interpreted as that ventricle that functions as the arterial ventricle. In corrected transposition this ventricle has the anatomical characteristics of a right ventricle and is usually left-sided. From such "left" ventricles both great vessels may arise, as in 2 of our 3 cases and in the case of Fragoyannis and Kardalinos (1962). To avoid confusion, however, the terms left-sided or right-sided ventricle have been used when referring to inverted ventricles.

It will be recalled that the features of anatomical inversion of the atrio-ventricular valves and of the ventricles in corrected transposition include what may be termed "pathological inversion" (inversion of pathological features). That is, as a structure of the normal heart is inverted in corrected transposition, so anomalies peculiar to a given structure may be seen in the contralateral side of the heart if corrected transposition is present. For example, while Ebstein's malformation occurs in the tricuspid (venous) valve of normal hearts, the same malformation occurs on the arterial side in corrected transposition. Also, as sub-aortic stenosis occurs in the left (arterial) ventricle of normal hearts, anatomically comparable types of subpulmonary stenosis occur in the right-sided (venous) ventricle in corrected transposition.

An additional example of so-called "pathological inversion" is exemplified by our cases. In the normal heart the ventricle with the infundibulum (right ventricle) may give rise to both great vessels. In our cases the ventricle with the infundibulum gave rise to both great vessels but, being inverted, this ventricle functioned as the arterial one. In two of our cases and in the one described by Fragoyannis and Kardalinos this ventricle was left-sided. In our third case (with mirror-image dextrocardia) this ventricle was on the right. 
It is possible that a lack of awareness of this transposition by clinicians and pathologists is responsible for the apparent rarity of the condition. Its clinical recognition is difficult since it so closely resembles corrected transposition of the great vessels. In each of the three cases reported here and in the case of Fragoyannis and Kardalinos, the diagnosis was made at necropsy. The most important clinical tool in the differentiation of this entity from corrected transposition is angiocardiography made selectively from the arterial ventricle. Cyanotic congenital cardiac disease with agenesis of the spleen should also alert the clinician to the possible presence of this malformation.

\section{SUMMARY}

The pathological and clinical features are presented in three children with the rare developmental anomaly, origin of both great vessels from the inverted arterial ventricle. In two instances, this ventricle was left-sided, while in the case with mirror-image dextrocardia, it was on the right.

In each case the pathological features were similar to corrected transposition except that both great vessels took origin from the inverted arterial ventricle.

Except for the angiocardiographic features, the clinical findings were of little value in establishing a definitive diagnosis. Selective angiocardiography from the arterial ventricle is the procedure of choice in establishing the diagnosis. The cardiac anomaly described may also be suspected when cyanotic congenital cardiac disease is associated with congenital absence of the spleen, a syndrome found in each of the three cases presented.

\section{REFERENCES}

Fragoyannis, S., and Kardalinos, A. (1962). Transposition of the great vessels, both arising from the left ventricle (juxtaposition of pulmonary artery). Amer. J. Cardiol., 10, 601 .

Grant, R. P. (1962). The morphogenesis of transposition of the great vessels. Circulation, $26,819$.

Neufeld, H. N., DuShane, J. W., and Edwards, J. E. (1961). Origin of both great vessels from the right ventricle. II. With pulmonary stenosis. Circulation, 23, 603.

$\longrightarrow$ - Wood, E. H., Kirklin, J. W., and Edwards, J. E. (1961). Origin of both great vessels from the right ventricle. I. Without pulmonary stenosis. Circulation, 23, 399

Ruttenberg, H. D., Neufeld, H. N., Lucas, R. V., Jr., Carey, L. S., Adams, P., Jr., Anderson, R. C., and Edwards, J. E. (1964). Syndrome of congenital cardiac disease with asplenia. Distinction from other forms of congenital cyanotic cardiac disease. Amer. J. Cardiol., 13, 387.

Schiebler, G. L., Edwards, J. E., Burchell, H. B., DuShane, J. W., Ongley, P. A., and Wood, E. H. (1961). Congenital corrected transposition of the great vessels: A study of 33 cases. Pediatrics, 27, 851 .

Spitzer, A. (1951). The Architecture of Normal and Malformed Hearts: A Phylogenetic Theory of Their Development. With a Summary and Analysis of the Theory by M. Lev, and A. Vass, p. 145. Charles C. Thomas, Springfield, Illinois.

Witham, A. C. (1957). Double outlet right ventricle. A partial transposition complex. Amer. Heart J., 53, 928. 